\title{
Role of Nanotechnology in Cancer Diagnostics
}

\section{Giuseppe Toffoli* and Flavio Rizzolio \\ Centro di Riferimento Oncologico-CRO-National Cancer Institute, Experimental and Clinical Pharmacology Unit, Aviano, Pordenone, Italy}

Cancer is a high heterogeneous disease difficult to understand and cure. Many historically classified tumors are now being revisited in order to better define the therapeutic strategy [1]. For example, the recent molecular classification of breast and ovarian cancers suggests a different pathological taxonomy to highlight the similarity with other types of cancers that originate from different organs [2]. On the other hand, detailed knowledge of the mechanisms behind the tumors can offer common clinical solutions for what is now a different pathology. The Cancer Genome Atlas Research Network (CGARN) has defined at molecular level a high similarity between basal like breast cancer and serious ovarian cancer which includes widespread genomic instability, gene alterations, and mRNA expression implying common biomarkers and therapeutic targets [3].

If the identification of the molecular targets is the first step in the chain for the cure of patients, it is imperative to rapidly translate all the bench information into clinics. There is a common agreement that early diagnosis is among the most promising approaches. The identification of biomarkers that can anticipate the development of tumors or can predict the response to a pharmacological treatment has the potential to dramatically alter the course of the disease [4].

In US, the National Cancer Insitute (NCI) has recognized that nanotechnology in various disciplines including physical sciences, engineering, physics, and chemistry has developed new materials and devices with exciting and promising approaches to address cancer problems. In September 2004, the Alliance for Nanotechnology in Cancer was created with the goal of translating and delivering innovative and clinically relevant technologies for cancer prevention, diagnosis, and treatment with the help of private sector partners (Cancer Nanotechnology plan).

The development of nanotechnology for the detection and translation of diagnostic biomarkers in clinical practice has many shortcomings to overcome, that can be summarized in two main categories: the quality of samples (Biobanking) and technological approaches.

The biospecimen quality is a critical issue that has recently received much attention since most of the current studies are at the international level and it is difficult to meet standard criteria. The CGARN had many difficulties in analyzing an adequate number of high-quality and well-annotated biospecimens [5]. Initiatives such as the Biospecimen Research Network Symposia and the Biospecimen Research database are fundamental to ensuring standard procedures and better pre-analytical methods are carried out. Standard Operating Procedures (SOP) that focus on infrastructure and specimen handling, data management, and ethical/social issues are currently in development (ISBER, IARC etc.) and will help to increase efficiency, reliability, and sustain international collaborations [6]. The advent of new nanotechnologies will further increase the necessity of developing prospective biobanks that meet the investigator's specific requirements. Variables such as the type of sensors, materials, revelator, and biological targets will require innovative biobanking strategies.

A vast number of new technologies are being developed in the diagnostic arena and the list of lab-on-a-chip Point-Of-Care Devices
(POC) for multi-analyte analysis is expanding [7]. A biosensor with POC characteristics should be easy to use and inexpensive while offering fast and robust multiplex service that can be utilized at home or in a physician's office. A pioneering POC was the immunoassay to measure urine hCG during pregnancy. The instrument is based on a lateral-flow on absorbent strip where the analyte is visualized with a coloured antibody in a fixed position and can be integrated with a digital readout of binding [8].

A modern biosensor is composed of recognition and transducer elements. As recognition element, most biomarker biosensors are antibody based, which have high sensitive and specificity. The limit is the short stability time and the need to refrigerate the device. For this reason, artificial receptors have been developed. Peptides, aptamers, binding proteins, and Molecular Imprinted Polymers (MIPs) are among the others successfully applied to the detection of different molecules [9]. In particular, MIPs are of outstanding interest since they are characterized by long-term stability and by the ability to perform well under harsh conditions, such as high temperatures, extreme $\mathrm{pH}$ values, and organic solvents. Functional and cross linking monomers are copolymerized in the presence of the target molecule, as such the specific functional groups and are held in position forming a three dimensional cavity that complements the shape, structure, and functional groups of the original template that can recognize and rebind the target with high specificity [10].

The main transducer signals fall under the category of electrochemical, optical, and mass change detectors. Electrochemical and optical devices are widely used in different fields and many cancer biomarkers such as PSA, CA125, and CEA can be measured [11]. A forerunner is the glucose biosensor that has been widely used to measure glucose at home in diabetic patients. Mass sensitive transducer quartz crystal microbalances have also been applied for cancer biomarker detection and a very recent pillar technology is developing with the promise of being a rapid test with a very high sensitivity [12]. In the near future, we will be able to measure biomarker concentrations on a device such as a smart phone as is exemplified in the lab-onchip SmartBioPhone device, which can be used in four point of care applications: environmental, food, cancer, and drug monitoring [13].

If the technology can be applied in the form of new low-cost devices, a wide-screening program could be realized thus improving and helping the health of the entire population.

*Corresponding author: Dr. Giuseppe Toffoli, Director of Experimental and Clinical Pharmacology Unit, Via Franco Gallini, 233081, Aviano, Pordenone, Italy, Tel: +39-0434-659-612; Fax: +39-0434-569-799; E-mail: gtoffoli@cro.it

Received January 31, 2013; Accepted February 11, 2013; Published February 22, 2013

Citation: Toffoli G, Rizzolio F (2013) Role of Nanotechnology in Cancer Diagnostics. J Carcinogene Mutagene 4: 135. doi:10.4172/2157-2518.1000135

Copyright: ( $\odot 2013$ Toffoli G, et al. This is an open-access article distributed under the terms of the Creative Commons Attribution License, which permits unrestricted use, distribution, and reproduction in any medium, provided the original author and source are credited. 


\section{Acknowledgments}

We thank Mr. Robert Fratamico for his assistance in copyediting the manuscript. Special Program Molecular Clinical Oncology, $5 \times 1000$, (No. 12214), European Research Council, Programme "Ideas", Proposal No 269051, Italian Ministry of Education MIUR (FIRB prot. RBAP11ETKA). The authors declare no conflict of interest.

\section{References}

1. Nicolin V, Bareggi R, Narducci P (2010) New Targeted Therapies Against Breast Cancer. J Carcinogene Mutagene 1: 110.

2. Vaughan S, Coward JI, Bast RC, Berchuck A, Berek JS, et al. (2011) Rethinking ovarian cancer: recommendations for improving outcomes. Nature reviews Cancer 11: 719-725.

3. The Cancer Genome Atlas Network (2012) Comprehensive molecular portraits of human breast tumours. Nature 490: 61-70.

4. Rusling JF, Kumar C V, Gutkind JS, Patel V (2010) Measurement of biomarker proteins for point-of-care early detection and monitoring of cancer. Analyst 135 : 2496-2511.

5. Blow N (2009) Biobanking: freezer burn. Nature Methods 6: 173-178.

6. Hewitt RE (2011) Biobanking: the foundation of personalized medicine. Curr opin oncol 23: 112-119.
7. Ahn CH, Choi J-W, Beaucage G, Nevin J, Lee J-B et al. (2004) Disposable Smart Lab on a Chip for Point-of-Care Clinical Diagnostics. Proceedings of the IEEE 92: 154-173.

8. Holford TR, Davis F, Higson SP, (2012) Recent trends in antibody based sensors. Biosens bioelectron 34: 12-24.

9. Tothill IE (2009) Biosensors for cancer markers diagnosis. Semin Cell Dev Bio 20: 55-62.

10. Sharma PS, D'Souza F, Kutner W (2012) Molecular imprinting for selective chemical sensing of hazardous compounds and drugs of abuse. TrAC Trends in Analytical Chemistry 34: 59-77.

11. Chikkaveeraiah BV, Soldà A, Choudhary D, Maran F, Rusling JF, et al. (2012) Ultrasensitive nanostructured immunosensor for stem and carcinoma cel pluripotency gatekeeper protein NANOG. Nanomedicine (Lond) 7: 957-965.

12. Melli M, Scoles G, Lazzarino M (2011) Fast detection of biomolecules in diffusion-limited regime using micromechanical pillars. ACS nano 5: 79287935.

13. Ruano-López JM, Agirregabiria M, Olabarria G, Verdoy D, Bang DD, et al. (2009) The SmartBioPhone, a point of care vision under development through two European projects: OPTOLABCARD and LABONFOIL. Lab chip 9: 14951499 